\title{
Research on Modeling and Simulation of Power Electronic Circuits Based on MATLAB
}

\author{
Quan Guo ${ }^{1, ~}{ }^{*}$, Kun $\mathrm{Xu}^{2}$,Fang Yi Sun ${ }^{3}$, Xue Qian $\mathrm{Li}^{4}$ \\ ${ }^{1}$ Military Simulation Technology Institute. Aviation University of Air Force, Ji lin province,Chang \\ chun city, China \\ ${ }^{2}$ Military Simulation Technology Institute. Aviation University of Air Force, Ji lin province,Chang \\ chun city, China \\ ${ }^{3}$ Military Simulation Technology Institute. Aviation University of Air Force, Ji lin province,Chang \\ chun city, China \\ ${ }^{4} 94565$ Army 75 Element,Anhui province, Beng Bu city,China \\ "baishikele15103@163.com
}

Keywords: Power electronic circuits, Modeling, Simulation

\begin{abstract}
. the example of three-phase bridge controlled rectifying circuit discussed the method of the utilization of MATLAB SIMULINK on the modeling and simulation of power electronic circuits, and gave the simulation result waveform, verifying the convenience, intuition, high-efficiency, high speed, genuineness and accuracy of this method. This method can also make modeling and simulation on quite complex circuits, power electronic conversion system, and electric drive automatic control system. The systematic modeling is very similar to the design process of the actual system. Users can get the simulation results of the system quickly without programming and the deduction of mathematical models of circuits and systems. The analysis on the simulation results can transform the system structure or modify relevant parameters in order to make the system get the required results and performance, which can greatly accelerate the analysis or design process of the system.
\end{abstract}

\section{Introduction}

In the power electronic circuits, such as the design process of converter device, it needs to verify the reasonability and effects of the initial scheme (circuits) and preferences of relevant elements. If it is tested through experiments, the designed system should be installed by elements for debugging and testing again. If it couldn't meet the requirement, it should change the elements, or even redesign, install, and debug. Satisfying results can be obtained over and over again. It not only wastes large quantity of manpower and material resources, but also lowers the design efficiency with big cost and long cycle.

The simulation test with the adoption of computers can greatly save the cost, improve the design efficiency, and shorten the design cycle. But it can be realized via other advanced computer languages (such as C language, BASIC language or emulation). As for power converter circuit, the high-power switching element switch convert current commutation dynamic process is very complex as the transition process is one by one that another new one will start while there is an unfinished one. In order to analyze output voltage and current (with inductive load) waveform, especially the size and the shape of the spike voltage which should be borne in the shutoff of the high-power switch tube, which is the protection effect of the RC protection circuit, it should build the mathematical model of the equivalent circuits. While such mathematical model is very complex that even it is built, the true simulation results which are obtained from the computer programming also need a large number of time and efforts on programming and debugging. While with the adoption of MATLAB/SIMULINK visualization, graphical simulation environment is intuitional, easy, convenient, high-efficient, fast, true and accurate for the modeling and simulation of electronic circuits. 
This paper takes the example of three-phase bridge controlled rectifying circuit as example, introducing the method of simulation modeling and method of power electronic circuits with the utilization of MAT-LAB/SIMULINK, and giving the simulation result waveform.

The power system toolbox and the visual graphic simulation function of simulation element introduction in SIMULINK is conducted in the environment of SIMULINK. After the entry of MATLAB system, open module library browser window, and double-click the left mouse button on the Power System Blocks, and then it appears power system toolbox module library. It includes Connectors, Electrical Sources, Elements, Extra Library, Machines, Measurements and Power Electronics. These module libraries contain most common used power system component modules. With the utilization of these library modules and other library modules, users can easily and intuitionally build various systematic modelings and simulations.

\section{The Building of Three-Phase Bridge Controlled Rectifying Circuit Simulation Model}

Open the new model window, drag the needed element modules from the module libraries into new model windows and rename them, and connect various modules into simulation models after setting relevant parameters.

Three-phase alternating current power supplies A, B and C are set as $220 \mathrm{~V}, 50 \mathrm{~Hz}$ with phase difference of $120^{\circ}$;

$\mathrm{La}, \mathrm{Lb}$ and Lc are equivalent inductance of three-phase power, setting as $5 \mathrm{mH}$;

The parameter of thyristor three-phase controlled rectifier bridge model is set as Thyristoron-state resistance $0.01 \Psi$, Thyristoron-state inductance $10 \mu \mathrm{H}$, Snubberresistance $2 \mathrm{~K} \Psi$ and Snubber capacitance) $4.7 \mu \mathrm{F}$;

Key2 Three-Phase Bridge Controlled Rectifying Circuit Simulation Model.The generating module of three-phase bridge controlled rectifier bridge trigger pulse is set as double pulsing with pulse width of $10^{\circ}$;

Set up synchronous voltage signal measurement conversion module and seal into subsystem TB;

$\mathrm{R}-\mathrm{L}$ load module parameters is set as $220 \mathrm{~V}, 50 \mathrm{~Hz}, 1000 \mathrm{~W}, 1000 \mathrm{Var}$;

3.7 Set up output voltage vl, cathode assembly three thyristor currents id, and thyristor voltage Uak to test display module.

\section{Simulation Operation and Simulation Result Waveform Analysis}

First double-click the Step module and set the alphade as $30^{\circ}$, and then click the Simulation button in the drop-down menu, and select simulation parameters order to set relevant simulation parameters. Set the Stop time as $0.08 \mathrm{sec}$. The simulation algorithm is selected as Variablestep integration algorithm function ode23s (Runge-Kutta method). Other parameters can be selected as default values. Then click to start simulation button (black angel), which will start the simulation. Double-click each display module, and then it will show its signal waveform. Keyis the voltage Uak(V) waveform among cathode assembly three thyristor AK. Each thyristor $30^{\circ}$ bears forward voltage. Breakover $120^{\circ}$ bears backward voltage at the rest of the time. Key 4 is the waveform of cathode assembly three thyristor currents id, which clearly shows the current commutation process. Key5 is waveforms of the output current id (A) and output voltage vl(V), clearly showing the current increasing process from 0 to a stable value, and the effect of the current size on thyristor commutation process. The simulation after changing each parameter setting cannot get the simulation waveforms of different parameters, thus analyzing the influence of each parameter on system. It provides the determination of system design parameter a scientific and easy method. Change the setting of each parameter until obtaining the satisfying results. 


\section{Modeling Language}

Modeling language provides a way to construct blocks with multiple inputs and multiple outputs. You can use these blocks by the block diagram.

A commercially available Pascal compiler compiles the modeling language, where the user has full advantage of the syntax of the compiler. The compiler generates fast executable code which the computer can execute directly. The compiler provides keywords such as; for, while do, repeat until loops, case statements and if-then program flow structures.

You can implement a control as an algorithm straight forward in the modeling language. This enables fast building and debugging of control functions, without translating the control algorithm into a block diagram first.

You can define the equations of components directly in Pascal. To model differential equations, for example

$$
\frac{d x}{d t}=[i-\sin (x)]^{2}
$$

you can use the following statements in Pascal.

$$
\operatorname{dxdt}[1]:=\operatorname{SQR}(\mathrm{i}-\sin (\mathrm{x}[1])) \text {; }
$$

CASPOC performs the integration of $\mathrm{x}[1]$ using fourth order Runge Kutta.

\section{Conclusion}

It can be seen from the above system simulation results that the system modeling and simulation results with the utilization of MATLAB/SIMULINK has reality and high credibility. This method can make modeling and simulation on complex circuits, power electronic converter system, and electric drive automatic control system. The systematic modeling is very similar to the design process of the actual system. Users can get the simulation results of the system quickly without programming and the deduction of mathematical models of circuits and systems. The analysis on the simulation results can improve the system structure or modify relevant parameters in order to make the system get the required results and performance, which can greatly accelerate the analysis or design process of the system.

\section{References}

[1] G.A. Franz, "Multilevel simulation tools for power converters", IEEE APEC CH2853-0/90/0000-0629, 1990.

[2] C-W. Ho, A.E. Ruehli, P.A. Brennan, "The modified nodal approach to network analysis", IEEE Transactions on Circuits and Systems, Vol CAS-22, No 6, June 1975.

[3] C.J. Hsiao, R.B. Ridley, H. Naitoh, F.C. Lee, "Circuit oriented discrete-time modeling and simulation for switching converters", IEEE 0275-9306/87/0000-0167, 1987.

[4] A.F. Schwarz, "Computer-aided design of microelectronic circuits and systems, Vol 1", Academic press 1987.

[5] "CASPOC User's manual", Simulation Research, P.O.Box 397, 2400 AJ, Alphen a/d Rijn, The Netherlands. Telephone : +31 172092353

[6] H. Polinder, N.H.M. Hofmeester, L.J.J. Offringa, W. Deleroi, "Cycloconverter for high speed permanent magnet generator units", Proceedings EPE Brighton, 1993.

[7] P. Bauer, J.B. Klaassens, "A novel control principle for parallel resonant voltage link converters", Proc. IEEE IAS annual Meeting, Huston, October 1992. 
[8] P.J. van Duijsen, "Multilevel modeling and simulation of electronic circuits and systems", Proceedings PE\&ED Košice, CSFR 1992.

[9] P.J. van Duijsen, "Multilevel modeling and simulation of power electronic systems", Proceedings EPE Brighton, England 1993.

[10]P.O. Lauritzen, C.L. Ma, "A simple diode model with reverse recovery", IEEE Transactions on Power Electronics, Vol 6 No 2, April 1991.

[11]P.J. van Duijsen, "MULTILEVEL MODELING AND SIMULATION OF POWER ELECTRONIC CONVERTERS AND DRIVE SYSTEMS", Power Electronics and Applications, 1993, 347 - 352 vol.4, Sep 1993. 